\title{
Mathematical modelling using improved SIR model with more realistic assumptions
}

\author{
Hamid A. Adamu, Murtala Muhammad, Abdullahi M.Jingi, Mahmud A. Usman
}

\begin{abstract}
David and Lang developed a mathematical model (SIR) i.e Susceptible-Infective-Recovered, for the spread of infectious in a given population over a time. The model gives a reasonable and sound results. However, some more realistic factors have not been accounted for in their model. These realistic assumptions will give better understanding of the modelling epidemics if included. Therefore, this paper is proposed to include some of these assumptions to improve the SIR model. We provide a number of contributions by incorporating the assumptions to enhance the analysis of the spread of diseases under different conditions. We have classified these conditions into four and provide experiments to each of the condition. We further provide a numerical scheme for the modified model and simulated the scheme using Visual Basic (VB) to test the model's accuracy. The results show that the modified model proved to be more efficient than the existing SIR model. However, due to the inclusion of new assumptions to the existing model, the modified model shows faster decrease in infected population and high recovery rate than the existing SIR model. Moreover, we also investigate the reproductive ration, stability analysis, equilibrium points of the model, the Jacobean and the eigenvalues of the model.
\end{abstract}

Index Terms - Mathematical model, SIR model, Epidemiology, endemic

\section{INTRODUCTION}

Infectious diseases are those diseases that can be transmitted from person to person or from organism to organism and are caused by microbial agents. Examples of such these include common cold, cholera, chicken pox etc. However, when a disease spreads in a given population, it splits the population into three classes (Martcheva, 2015). The class of individuals who are healthy but can contract the disease (susceptible-S), the class of individuals who have contracted the disease and are now sick (infected-I). However, it is assumed that the infected individuals are also infectious, and the last class of individuals is those who have recovered and cannot contract the disease again (recovered-R). The number of individuals in each of these classes changes with time. That's they are functions of time $t$ and the total population size $(\mathrm{N})$ is the sum of the sizes of these three classes:

$N(t)=S(t)+I(t)+R(t)$.

The extent to which a disease exists or spreads in a given population depends on the degree and frequency of contact between susceptible individuals and disease agents (Lucas and Gilles, 1982).The impact of infectious disease on human

H.A Adamu School of Computing Science and Engineering, University of Salford, Manchester, UK

M. Muhammad, School of Computing Science and Engineering, University of Salford, Manchester, UK

Abdullahi .M.Jingi, Adamawa State University, Mubi-Nigeria

M. A. Usman School of Computing Science and Engineering, University of Salford, Manchester, UK. and animals is enormous both in terms of suffering, social and economic consequences. Mathematical modeling is an essential tool in studying a diverse range of such diseases. In fact, the threat of infectious diseases seems to have replaced nuclear war in in this generation (Bryan, Stefan and Mark, 2005)

When new diseases enter new population, we begin to envisage different kinds of outcomes such as; the disease could quickly die out, it could remain in the population more or less stable level, perhaps; "settling down" after a major outbreak (i.e become endemic), it could circle in incidence, causing periodic epidemic or epidemic could come and go at more or less intervals, perhaps exhibiting "chaotic" behavior. Mathematical models may give us real insight on such outcome (Epidemiology sample activity, 2003). The basic aims in studying the spread of infectious diseases in time and space is to gain a better understanding of the transmission mechanism and those features that are most essential in their spread, so as to enable prediction to be made, determined and evaluation control strategies (Bryan, Stefan and Mark 2005). Recently, mathematical modeling has emerged as a key tool in the studying diversity and control of infectious diseases, allowing virtual epidemics to be run, testing the effects of different assumptions or possible interventions (Henry, 2006). This paper is to provide remedy for some of the deficiencies in then work by David and Lang (2001), by incorporating some more realistic assumptions so as to develop a better mathematical model of the dynamic of infectious disease and compare between existing model and the modified model.

In this paper, we provide a number of contributions to the understanding of the role interaction and economic activity in the spread of diseases. The paper improves on the literature in epidemiology, which has developed models of disease diffusions dating back to Kermack and McKendrick (1927) by providing some factors that are more realistic factors that not being accounted for in the SIR model.

\section{STATEMENT OF THE PROBLEM}

David and Lang (2001) developed a mathematical model (SIR) i.e Susceptible-Infective-Recovered for the spread of infectious in a given population over a time. However, there are some realistic factors that not being accounted for in this model and this paper is proposed to address some of these factors. These factors include: -

- The effects of birth and death due to other causes were not taken into consideration.

- The infected individuals that die due to the disease were not considered

- The general health and nutrition status of the populations, which may have a positive effect on the likelihood of epidemic occurring, were not considered. 


\section{LITERATURE REVIEW}

Infection diseases such as malaria, aids and cholera continue to claim millions of lives around world (Busenberg \& Cooke, 1993). However, global eradication programs of infection diseases have been implemented for many years with considerable success (Howard Weiss,2013). He further illustrates how the model helps in understanding a theoretical analysis for public health interventions.

Application of mathematical modeling to the spread of epidemic has a long history and was initiated by Daniel Bernoulli who worked on the effects of cow-pox inoculation on the spread of small pox in 1760 (keeling, Woolhouse, many Davies and Greenfell 2003). Diseases are ubiquitous part of human life. Many, such as the common cold have minor symptoms and are purely an annoyance; but others such as Ebola or aids fill us with dread. From prehistory to the present day, diseases have a source of fear and superstition. Over past one hundred years, mathematics has been used to understand and predict the spread of disease, relating important public health question to basic infection parameters (keeling, 2001)

Meghan (1998) developed a simple mathematical model for number of people infected with an infection disease such as chicken pox in a closed population. His study showed that, if the parameter governing the spread of the disease is positive, the disease will spread throughout the population and eradication is not possible. On the other hand, if the parameter is negative, the chicken pox dies out in the population.

Kermack and Mckendrick (1927), Proposed an SIR model to explain the rapid rise and fall in number of infected patients observed in epidemics such as cholera and Bubonic plague in London. They assumed an affixed population size, incubation period of the infectious agents is instantaneous and the duration of the infectivity is the same as the length of the diseases. The research revealed that, an epidemic would occur if and only if the initial fraction of the susceptible exceeds the reproductive ration (Ro). Looking at the long-term behavior of their model, they further predicted the proportions of susceptible who would escape the infection. The results showed that, if the reproductive ratio Ro is small then more people would escape the infection and vice-versa.

Troy (2005) introduced a mathematical model that describes the data of "excess" pneumonia-influenza deaths adopted from David and Lang (2001). He assumed that, in a fixed homogeneous population the number of excess deaths is proportional to the number of incidence of the disease over the time. The work showed that, if Ro<1 the disease dies out with time. He further discovered that, the epidemic that slowly kills people can be far more dangerous to the population than the disease that kills people quickly. This result is seen in the epidemic such as HIV/AIDS and Bubonic plague. According to him, if a disease removes its carriers quickly, then the disease is not likely to have a long life span. Keeling (2001) estimated the reproductive ratio (Ro) of some well-known infectious diseases; malaria $\mathrm{Ro} \approx 100$, measles $\mathrm{Ro} \approx 18$, small pox $\mathrm{Ro} \approx 4$ and AIDS Ro $\approx 5$. His research showed that, infectious diseases with higher values Ro are harder to control than those with lower values of Ro. According to him, it is because of this fact that vaccination has allowed us to completely eradicate small pox $(\mathrm{Ro} \approx 4)$ and it is very hard to control malaria $(\mathrm{Ro} \approx 100)$. Hufnagel, Brockmann and Geisel (2003), developed a probabilistic model which was based on the mathematical model (SIR) to describe worldwide spread infectious disease such as Severe Acute Respiratory Syndrome (SARS).

The model combines a stochastic local infection dynamics among individuals with stochastic transport in a worldwide network, taking into account national and international civil aviation traffic. The model was used to predict the spread of SARS for ninety days after the initial outbreak. In Hong Kong 2003. The results of the simulation were in remarkable agreement with the worldwide spread of SARS as reported by World Health Organization (WHO). They further suggested that based on the results, the model can be used to predict the worldwide spread of future infectious diseases and identify threatened region in advance. With respect to control, the simulation showed that a quick and focused reaction is essential to inhibiting the global spread of epidemics. David and Lang (2001) developed the mathematical model (SIR) for spread of infectious diseases such as Hong Kong flu in New York in 2001. The assume the total population to be fixed during the flu epidemic by ignoring births, immigrations and deaths due to non-flu causes, also assumed a completely homogeneous population with no age spatial or social structure. They study revealed that, if the contact number of the disease is small enough then no epidemic can develop and vice-versa.

\subsection{The existing model}

The existing SIR model is given as

$S^{\prime}=-g S(t), S(0)=S_{0}$

$I^{y}=(g S(t)-k), I(0)=I_{0}$

$R^{\prime}=k I(t), R(0)=R_{0}$ where

$\mathrm{S}(\mathrm{t})=$ number of susceptible at time $\mathrm{t}$.

$I(t)=$ number of infected at time $t$.

$\mathrm{R}(\mathrm{t})=$ number of recovered at time $\mathrm{t}$.

$\mathrm{N}(\mathrm{t})=$ total population at time $\mathrm{t}$.

$\mathrm{g}=$ infectious rate of the disease.

$\mathrm{k}=$ recovery rate of the disease.

$R_{0}=$ reproductive ratio of the disease.

The model presented in this work is basically a SIR model. This model classifies individuals in a given population into susceptible, infective and recovered. However, in this model, an individual potentially moves from susceptible pool to the infective pool when he/she meets an infected person. For instance, the contact for common cold may be walking within a few feet of an infected person that has recently coughed. Infectious individuals spread the disease to the susceptible and remain in the infectious pool for some period of time (infectious period), before moving into recovered pool.

\section{Methodology}

The mathematical modeling methodology in building the new model by modifying the existing SIR model through incorporating assumptions that are more realistic. The existing SIR model give good analysis epidemic diseases but considering more realistic factors to the model will give better results. The equilibrium and stability analysis of the model will be examined. The eigenvalues of the improved model have been analysed. The numerical scheme of the model will be implemented and the corresponding simulation will be executed to draw some interpretations. 
To formulate the model, we consider how those classes change over time and consider more assumptions. However, when a susceptible individual enters into contact with an infectious individual, that susceptible individual becomes infected with a certain probability and moves from the susceptible class into the infected class. The susceptible population decreases in a unit of time by all individuals who become infected in that time. At the same time, the class of infectives increases by the same number of newly infected individuals. The number of individuals who become infected per unit of time in epidemiology is called incidence, and the rate of change of the susceptible class is given by $S(t)=$ -incidence

\subsection{Model formulation}

As the first step in modeling process, we identify the independent and dependent variables. the independent variables is time $t$, measured in days months or years depending on the disease under study and the depend variables counts people in each of the groups, each as a function of time.

where; $S=S(t), I=I(t)$, number of infected individuals at time $t, R=R(t)$, number of recovered individuals at time $t$ and $N=N$ $(\mathrm{t})$, total population at time $\mathrm{t}$. Each individual in a population is in one of the three pools

Thus $\mathrm{S}(\mathrm{t})+\mathrm{R}(\mathrm{t})=\mathrm{N}(\mathrm{t})$

\subsection{Assumptions of the model}

In addition to the assumptions In David and Lang, we make some assumptions in order to build a more realistic model.

Basic SIR Models make the following assumptions:

(i) The rate at which the susceptible become infected is proportional to the infective and susceptible i.e $g S(t) I(t)>0$ is a constant parameter.

(ii) The rate of recruitment of the infective to the recovered pool is proportional to the infective that acquired good treatment i.e $k I(t), k>0$ is a constant parameter.

(iii) The population is completely homogenous and the incubation period of the disease is instantaneous.

(iv) We assume that, the common birth rate of the population is $b>0$ and the date rate due to natural causes in the population is $d>0$

(v) We assume that, all newly born individuals are born into the susceptible pool and individuals are equally likely to die to natural causes in any of the population pools.

(vi) We assume that, the infected individuals die at a rate $q>0$ due to the disease.

The first 3 assumptions are from the existing SIR model whereas, the last 2 are the new incorporated realistic assumptions

In summary we can conclude that;

- Individuals are born into the susceptible class.

- Susceptible individuals have never come into contact with the disease and are able to catch the disease, after which they move into the infected class.

- Infected individuals spread the disease to susceptible and remain in the infected class (the infected period) before moving into the recovered class.
- Individuals in the recovered class are assumed to be immune for life.

Finally, we make the simplifying assumption that the common birth rate and death rate of the population are equal so that, the population remains constant.

\subsection{Modified Model}

Since epidemiology is the study of the spread of diseases with the objective of tracing factors that contribute to their occurrence (Jiang D et al, 2011), we introduce more realistic assumptions to the existing model given in section (4.1). The modified model consists of a system of three first order ordinary differential equations that specify the rate of change of three categories of individuals in the population over time
$S^{\prime}=b-(g I(t)+d) S(t)$
$I^{\prime}=(g S(t)-k-d-q) I(t)$
$R^{\prime}=k I(t)-d R(t)$

To complete the model, each of the differential equation above is given an initial value, that is

$S^{\prime}=b-(g I(t)+d) S(t), \quad S(0)=S o$

$I^{\prime}=(g S(t)-k-d-q) I(t), I(0)=I o$

$R^{\prime}=k I(t)-d R(t), R(0)=R o$

where $\mathrm{b}=$ constant recruitment rate into the susceptible population.

$\mathrm{d}=$ natural death rate of the population.

$\mathrm{q}=$ date rate due to disease.

Ro $=$ reproductive ratio of the disease.

$$
\begin{aligned}
& s(t)=\frac{S(t)}{N(t)}, i(t)=\frac{I(t)}{N(t)}, r(t)=\frac{r(t)}{N(t)} \\
& \text { and } s(t)+i(t)+r(t)=1 .
\end{aligned}
$$

\subsection{Reproductive ration}

Considering (4.8.5), it follows that

$\frac{d I}{d t}=I(g S-k-d-q)$. However, to eradicate the disease in the host population the condition $\frac{d I}{d t}<0$ must hold. Implies that

$I(g S-k-d-q)<0$.

Therefore either $I<0$ or $(g S-k-d-q)<0$.

Clearly $\mathrm{I}<0$ is trivial for epidemic not to occur (eradication). Now consider;

$(\mathrm{gS}-\mathrm{k}-\mathrm{q})<0$ therefore, $\frac{g S}{k+d+q}<1$.

Let $R_{0}$ denote the reproductive ratio and So the initial value of the susceptible population and define $R_{0}$ as

$R_{0}=\frac{g S_{0}}{k+d+q} \quad$ (4.4.1) The reproductive ratio is the fundamental parameter governing disease dynamics in our model. Thus we have the following results.

(i) $R_{o}<1$ physically interpreted to mean that each person gets the disease will infect less than one person before recovering or dying, so the disease will peter out and eradication is possible $\frac{d I}{d t}<0$

(ii) $\mathrm{R}_{\mathrm{o}}>1$ physically interpreted to mean that each person who gets the disease will infect more than one person, so the epidemic will spread in the host population $\frac{d I}{d t}>0$. 


\subsection{Stability analysis of the model.}

To determine the stability points of the modified model, the Jacobian matrix of the system is obtained and evaluated at the equilibrium points. The eigenvalues of the Jacobian matrix is determined and is the stability points of the model.

\subsubsection{Asymptotic stability.}

Considering the modified model equations, these equations can also be written as;

$$
\begin{aligned}
& S^{y}=b-\left(g(I(t)+d) S(t)=f_{1}\right. \\
& I^{y}=\left(g(S(t)-k-d-q) I(t)=f_{2}\right. \\
& R^{y}=k I(t)-d R(t)=f_{3}
\end{aligned}
$$

where $f$ is the incidence rates and are monotonic and concave with respect to I to achieve threshold dynamics of epidemic models (Ba Y \& Mu X, 2018).

\subsection{Equilibrium of the model}

To evaluate the equilibrium points of the model, we set the RHS of ((4.5.1) to zero.

$$
S^{b}=b-g(I(t)+d) S(t)=0
$$$$
I^{y}=(g(S(t)-k-d-q) I(t)=0
$$

$R^{\prime}=k I(t)-d R(t)=0$

Considering $g(S(t)-k-d-q) I(t)=0$, it follows that either $I=0$ or $S(t)-k-d-q)=0$. However if $I=0$, we have $b-d S=0$, implies that $S=\frac{b}{a}$. Meanwhile, considering $k I(t)-d R(t)$ and putting $I=0$, it follows $-d R=0$. Similar results for $I=0$. Therefore, the equilibrium points of the model are $\left(\frac{b}{a}, 0,0\right)$ which is the Disease Free Equilibrium (DFE).

\subsection{The Jacobian matrix}

The Jacobian matrix of the modified model is

$$
I=\left[\begin{array}{lll}
\frac{\partial f_{1}}{\partial S} & \frac{\partial f_{1}}{\partial I} & \frac{\partial f_{1}}{\partial R} \\
\frac{\partial f_{2}}{\partial S} & \frac{\partial f_{2}}{\partial I} & \frac{\partial f_{2}}{\partial R} \\
\frac{\partial f_{3}}{\partial S} & \frac{\partial f_{3}}{\partial I} & \frac{\partial f_{3}}{\partial R}
\end{array}\right]
$$

Therefore, $l=\left[\begin{array}{ccc}-(g I+d) & -g S & 0 \\ g I & g s-(k+k+d) & 0 \\ 0 & 0 & -d\end{array}\right]$

The Jacobean evaluated at the DFE is obtained as

$$
I\left(\frac{b}{a}, 0,0\right)=\left[\begin{array}{ccc}
-d & -\frac{b g}{d} & 0 \\
0 & \frac{b g-(k+d+q) d-\lambda d}{d} & 0 \\
0 & k & -d
\end{array}\right]
$$

\subsection{The eigenvalues}

To determine the eigenvalues, we solve the equation $|J-\lambda I|=0$. Evaluating the equation give the eigenvalues as follows

$$
\lambda_{1}=\lambda_{2}=-d \text { and } \lambda_{3}=\frac{b g-(k+d+q) d}{d}
$$

Therefore, it can be clearly $\lambda_{1}$ and $\lambda_{2}$ satisfy the negativity requirements for stability because $d$ is a positive parameter. On the other hand, for $\lambda_{3}<0, b g-(k+d+q) d$. must be less than zero since $d>0$. Thus, $b g<(k+d+q) d$ which give

$g<\frac{(k+d+q)}{b}$

Therefore, the DFE is locally asymptotically stable, hence the disease eradication is possible under these conditions.

V. NUMERICAL SCHEME FOR THE MODEL EQUATIONS

Now considering the system of first order ordinary

$S_{n+1}=S_{n}\left(I-h\left(g I_{n}+d\right)\right)+h b, S(0)=S_{0}$

$I_{n+1}=I_{n}\left(I+h\left(g S_{n}-k-d-q\right)\right), I(0)=I_{0}$

$R_{n+1}=R_{n}(I-d h)+h K I_{n}, R(0)=R_{0}$

for $n=0,1,2, \ldots$

Differential equations that arise from the formulation of our model can be written as:

$S^{\prime}=b-(g I+d) S_{j} S(0)=S_{0}$

$I^{y}=(g S-k-d-q) I_{,} I(0)=I_{0}$

$R^{\prime}=k I-d R_{,} R(0)=R_{0}$

Using Euler's formula, we can write the system of equations as $S_{n+1}=S_{n}+h\left(S^{J}\right)_{n}$

$I_{n+1}=I_{n}+h\left(I^{n}\right)_{n}$

$R_{n+1}=R_{n}+h\left(R^{\prime}\right)_{n}$

From (5.1.2) we have

$\left(S^{\prime}\right)_{n}=b-\left(g I_{n}+d\right) S_{n}$

$\left(I^{\prime}\right)_{n}=\left(g S_{n}-k-d-q\right) I_{n}$

$\left(R^{\prime}\right)_{n}=K I_{n}-d R_{n}$

for $n=0,1,2, \ldots$

Therefore, substituting we obtain

$S_{n+1}=S_{n}+h\left(b-\left(g I_{n}+d\right) S_{n} S(0)=S_{0}\right.$

$I_{n+1}=I_{n}+h\left(g S_{n}-k-d-q\right) I_{n}, I(0)=I_{0}$

$R_{n+1}=R_{n}+h\left(k I_{n}-d R_{n}\right), R(0)=R_{0}$

for $n=0,1,2, \ldots$

\section{COMPUTER SIMULATION}

The numerical scheme developed in section 5.0 above is simulated using Visual Basic (VB) We have simulated both the existing model by David and Lang as well as our modified model. We have selected some initial values for the numerical experiments and obtained the results. However, the experiments are carried out in four stages as follows

i. We examine the dynamics of infectious disease under high transmission rate and high recovery rate.

ii. We examine the dynamics of the infectious disease under low transmission rate with high recovery rate.

iii. We study the dynamics of the infectious disease under high transmission rate and zero recovery rate.

iv. We examine what will happen to the host population, if there is no infection

\subsection{Discussion of results}

The discussions of the results for the four stages of the excitements are given as

i. Under high transmission rate and high recovery rate, the results of the model by David and Lang (2001) is displayed in figure 1(b). 


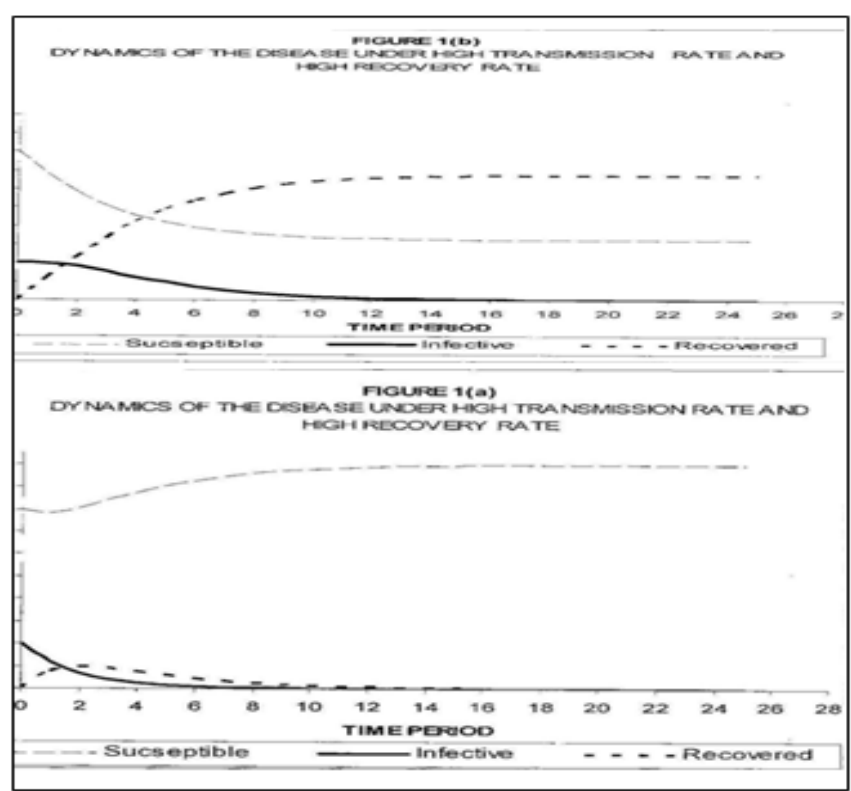

Figure 1(a) and (b): Dynamics of diseases under high transmission rate and high recovery rate

There is a decrease in proportions of the infected individuals due to good treatment (high recovery rate)as indicated on Figure 1 above. On the other hand, the results of the modified model is presented in figure 1(a) and it shows a faster decrease in the proportions of the infected individuals than the previous model. Therefore, the modified model shows faster decrease in infected population and this is due to the inclusion of new assumptions to the existing model. Moreover, this result agrees with the results of our reproductive ratio (Ro) in section (4.4). In this experiment $\mathrm{Ro} \approx 0.6$ which is less than 1 .

ii. Under low transmission rate with high recovery rate, the results shows that under high recovery rate (good treatment given to the infected), it takes longer period of time to eradicate the infection in the previous than the modified model. Thus, the modified model shows that the infection can be eradicated faster than the previous model as shown in Figure $2(\mathrm{a}))$ and agrees with reproductive ration $(\mathrm{Ro} \approx 0.12)$

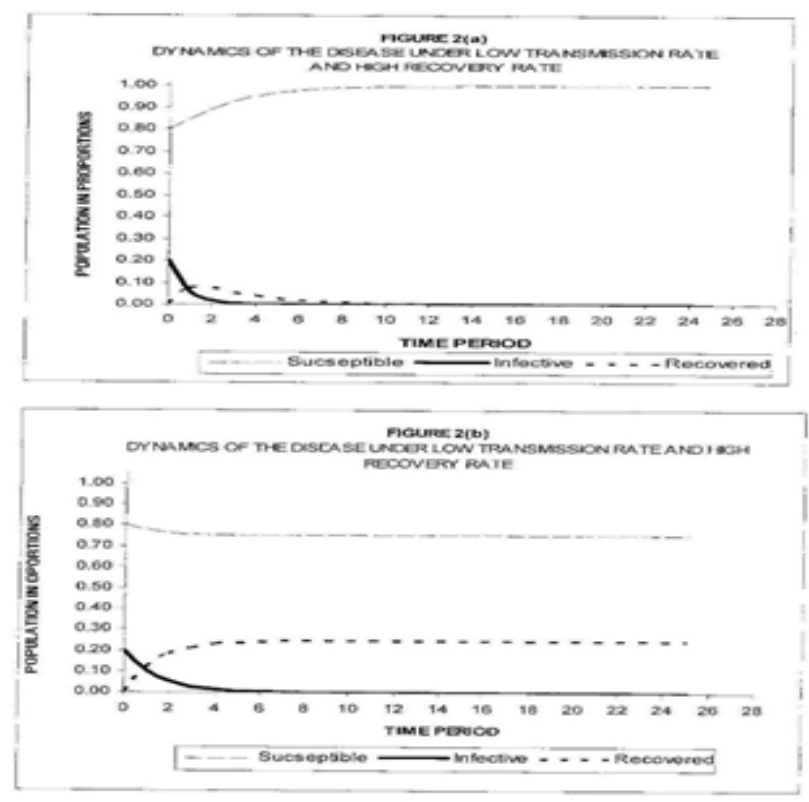

Figure 2 (a) and (b): Dynamics of the infectious disease under low transmission rate with high recovery rate. iii. The infectious disease under high transmission rate and zero recovery rate shows that the model by David and Lang reveals high rise in proportions of the infected individuals due to absence of treatment given to infected individuals. This leads to the extinction in proportions of the susceptible individuals as shown in Figure $3 \mathrm{~b}$. This is a clear indication that the existing model can only be used to describe spread of those diseases that persist for short period (i.e. epidemic) and does not account for recruitment rate into the susceptible pool. Whereas the results of the modified version clearly describe the dynamics of the infection that persists through the periods (i.e. become endemic). Here there is no extinction of the susceptible individuals since the recruitment rate into the susceptible pool has been considered. Therefore, the modified version of the model can be used to describe dynamics of both epidemic and endemic diseases. The persistence of the infection agrees with results of the reproductive ratio $(\mathrm{Ro} \approx 2.7)$ as shown on the result. .e. Ro> 1).

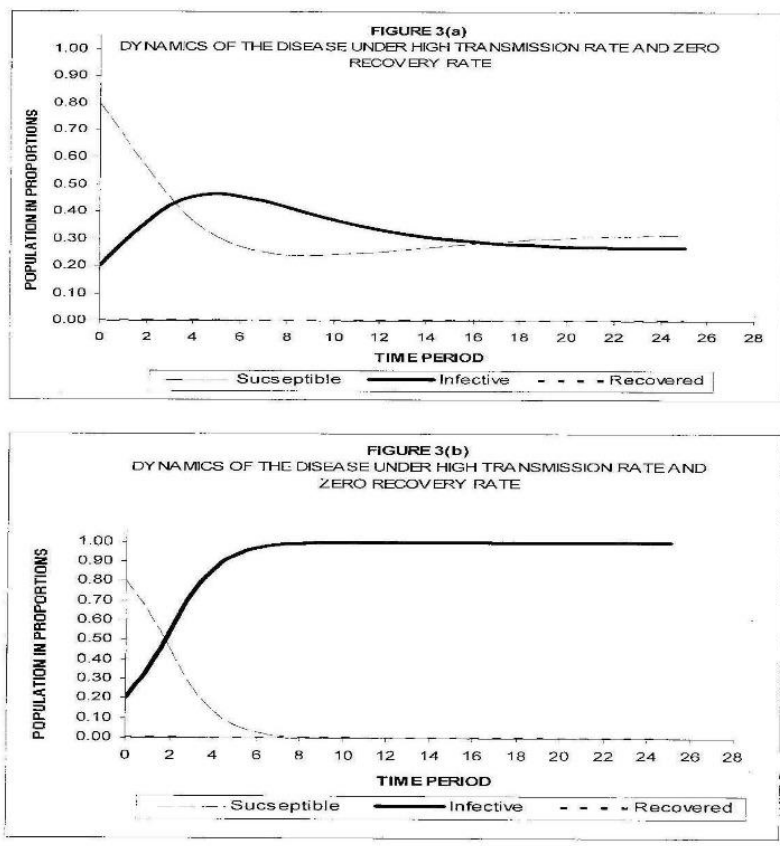

Figure 3 (a) and (b): The infectious disease under high transmission rate and zero recovery rate

iv. Without infection the host population would remain uninfected. However, this is naturally and obviously expected since both of the models do not account for migration.

\section{CONCLUSION}

The modified version of mathematical model proposed and studied in this paper been proved more practical than that by David and Lang, as it gives a better insight into the dynamics of infectious diseases and consequently enhances struggle against the spread of those diseases. However, it also revealed some remarkable result. Under high transmission rate disease spread in the host population but with massive effective treatment given to the infected individuals, the improved model shows a drastic reduction of length of time taken to achieve eradication. The modified version of the model can be used to study and describe both epidemic and endemic diseases unlike the model by David and Lang which can only study the spread of those diseases that persist for 
short period of time i.e. epidemic. This study also proved beyond doubt that, under low transmission rate infection spread less, therefore good medical care and effective treatment to the infected individuals dramatically lower the transmission rate and hence making eradication possible in finite time.

Based on the findings in this work, we recommend the modified version of the model for use by the epidemiologists as an appropriate tool in understanding how infectious diseases spreads, assessing the risks and evolving optimal control strategy. Finally, we recommend that quarantining the sick people would serve as a preventive measure towards inhibiting disease transmission and possibly lead to eradication.

\section{REFERENCE}

[1] Bailey N.T.J. (1982) Biomathematics of Malaria. New York Charles Griffm and company Limited.

[2] BryanT.G.Stefan, M.\& Mark,C.(2005) Mathematical modeling of Infectious. Diseases Dynamics and control. Institute for Mathematical Sciences, National University of Singapore Regional Emerging Diseases Intervention (REDI) Centre. Singapore.

[3] Busenberg, S. and Cooke, K. (1993) Vertical Transmitted Diseases-Models and Dynamics. New York: Springer and Verlag.

[4] David, S. \& Lang, M. (2001).The SIR Model of Infectious DiseasesJournal of Online Mathematics and its Applications. Mathematical Association of America.

[5] Epidemiology Sample Activity (2003) Modeling the spread of Diseases.

[6] Henry, N. (2006). Mathematical Modeling of Infectious Diseases. London: Wellcometrust.

[7] Hufnagel, L., Brockmann, D. \& Geisel, T. (2003) Forecast and control of Epidemic in a Globalize World. Oxford: Oxford University Press.

[8] Howard Weiss (2013). The SIR Model and the foundations of public health. volum 203, treball no. 3, 17 pp. ISSN: 1887-1097.

[9] Keeling, M. (2001). Mathematics of Diseases. Cambridge:Millennium Mathematics project Universiy of Cambridge.

[10] Kermack, W.O. \& Mckendrick, A.G. (1927) Kermack-Mckendrick model. http://mathworld. wolfram. com/kerrnack Mckendrickmodel.html

[11] Lucas, A.O. \& Gilles, H.M. (1982) A Short Textbook of Preventive Medicine for the Tropics. London: Hodder and Stoughton.

[12] Meghan, A.B. (1998) Epidemiology Model (Differential Equations) part 1

[13] Martcheva (2015) An Introduction to Mathematical Epidemiology,department of Mathematics, Univeraity of Florida. 